\title{
A CONNECTION BETWEEN REGULARITY AND DIRICHLET PROBLEMS FOR NON-DIVERGENCE ELLIPTIC EQUATIONS
}

\author{
JORGE RIVERA-NORIEGA
}

Abstract. We observe that a version of Poincaré's inequality for positive solutions to second order linear non-divergence form equations vanishing on a portion of the boundary, implies a natural connection between $L^{p}$ Dirichlet and $L^{q}$ Regularity problems for this type of equations.

Mathematics subject classification (2010): 35B09,35J25.

Keywords and phrases: Poincaré inequality, $L^{q}$ Regularity problem, $L^{p}$ Dirichlet problem, Reverse Hölder inequality.

\section{REFERENCES}

[1] P. Bauman, Positive solutions of elliptic equations in nondivergence form and their adjoints, Ark. Mat 22 (1984), 153-173.

[2] B. E. J. Dahlberg, On estimates of harmonic measure, Arch. Rat. Mech. Anal. 65 (1977), 272-288.

[3] - On the Poisson integral for Lipschitz and $C^{1}$ domains, Studia Math. 66 (1979), 13-24.

[4] On the absolute continuity of elliptic measure, Amer. J. of Math. 108 (1986), 1119-1138.

[5] M. Dindos, C. E. Kenig, and J. Pipher, BMO solvability and the $A_{\infty}$ condition for elliptic operators, J. Geom. Anal. 21 (2011), 78-95.

[6] M. Dindos and J. Kirsch, The regularity problem for elliptic operators with boundary data in HardySobolev space $H S^{1}$, Math. Res. Lett. 19 (2013), 699-717.

[7] M. Dindos, S. Petermichl, and J. Pipher, The $L^{p}$ dirichlet problem for second order elliptic operators and a p-adapted square function, J. Funct. Anal. 249 (2007), 372-392.

[8],$B M O$ solvability and the $A_{\infty}$ condition for second order parabolic operators, Annals de l'Institut Henri Poincare (C) Non Linear Analysis. doi: 10.1016/j.anihpc.2016.09.004 (2016).

[9] M. Dindos and T. Wall, The $L^{p}$ Dirichlet problem for second order, non-divergence form operators: solvability and perturbation results, J. Funct. Anal. 261 (2011), 1753-1774.

[10] L. Escauriaza and C. E. Kenig, Area Integral estimates for solutions and normalized adjoint solutions to nondivergence form elliptic equations, Ark. Mat. 31 (1993), 275-296.

[11] R. Fefferman, C. E. Kenig, and J. Pipher, The theory of weights and the Dirichlet problem for elliptic equations, Ann. of Math. 134 (1991), 65-124.

[12] S. Hofmann, J. M. Martell, and S. Mayboroda, Uniform rectifiability, Carleson measure estimates, and approximation of harmonic functions, Duke Math. J. 165 (2016), 2331-2389.

[13] D. Jerison and C. E. Kenig, Boundary behavior of harmonic functions in nontangentially accessible domains, Adv. in Math 146 (1982), 80-147.

[14] C. E. Kenig, Potential theory of non-divergence form elliptic equations, Dirichlet forms (Varenna 1992), Springer, Lecture Notes in Mathematics vol. 1563, 1993, pp. 89-128.

[15] C. E. Kenig, B. Kirchheim, J. Pipher, and T. Toro, Square functions and the $A_{\infty}$ property of elliptic measures, J. Geom. Anal. 16 (2016), 2383-2410.

[16] C. E. Kenig, H. Koch, J. Pipher, and T. Toro, A new approach to absolute continuity of elliptic measure with applications to non-symmetric equations, Adv. in Math. 153 (2000), 231-298.

[17] C. E. Kenig and J. Pipher, The Neumann problem for elliptic equations with non-smooth coefficients, Invent. Math. 113 (1993), 447-509.

[18] — The absolute continuity of elliptic measure revisited, J. Fourier Anal. Appl. 4 (1998), $463-$ 468. 
[19] - The Dirichlet problem for elliptic equations with drift terms, Publ. Mat 45 (2001), 199-217.

[20] C. Rios, The $L^{p}$ Dirichlet problem and nondivergence harmonic measure, Trans. Amer. Math. Soc. 355 (2003), 665-687.

[21] $L^{p}$ regularity of the Dirichlet problem for elliptic equations with singular drift, Publ. Mat. $\mathbf{5 0}$ (2006), 475-507.

[22] J. Rivera-Noriega, Absolute continuity of parabolic measure and area integral estimates in noncylindrical domains, Indiana Univ. Math. J. 52 (2003), 477-525.

[23] _ Perturbation and solvability of initial $L^{p}$ Dirichlet problems for parabolic equations over non-cylindrical domains, Canadian J. Math 66 (2014), 429-452.

[24] C. Sbordone and G. Zecca, The $L^{p}$ solvability of the Dirichlet problem for planar elliptic equations, J. Fourier Anal. Appl. 15 (2009), 871-903.

[25] Z. Shen, A relationship between the Dirichlet and Regularity problems for elliptic equations, Math. Res. Lett. 14 (2007), 205-213.

[26] P. Sjögren, On the adjoint of an elliptic linear differential operator and its potential theory, Ark. Mat 11 (1973), 153-165.

[27] G. Verchota, Layer Potentials and regularity for the Dirichlet problem for Laplace's equation in Lipschitz domains, J. Funct. Anal. 59 (1984), 572-611.

[28] M. Wuertz, The Implicit Function Theorem for Lipschitz Functions and Applications, Master's Thesis, University of Missouri-Columbia, May of 2008. 